\title{
A Qos Multicast Routing Protocol in WSN Network
}

\author{
Yang Wenzhong, Zhang Zhenyu \\ School of Information Science and Engineering Xinjiang University \\ URUMQI 830046, China \\ E-mial: ywz_xy@163.com
}

\begin{abstract}
The multicast information is usually transmitted through multiple intermediate nodes to reach the final destination node in multi-hop cognitive radio networks. There exist a lot of multicast routing protocols research, while these protocols based on traditional wireless networks are not suitable for WSN network. The question to be solved is in this paper: how to create a multicast routing path in multi-hop wireless WSN network, given a multicast routing QoS requirements, leads to the least bandwidth cosumption in the transmission process In this paper, a distributed multicast routing protocol is proposed to resolve the issue, the agreement not only completes the establishment of the routing process, but also conquers the reasonable scheduling of nodes in the routing transfer process. Experimental results show that the Qos multicast routing protocol can effectively reduce network bandwidth consumption, and increase the success rate of the response of the multicast request
\end{abstract}

Keywords- WSN network; Multicast Routing; Quality of service; Qos; Scheduling

\section{INTRODUCTION}

Wireless sensor network (WSN)[1-4] is widely considered as one of the most important technologies in the twenty-first century. because sensor nodes are powered by battery and it is often very difficult or even impossible to charge or recharge their batteries. QoS protocols in sensor networks have several applications including real time target racking in battle environments, emergent event triggering in monitoring applications etc.

Different unicast, multicast [6-10] implementation is transferred from point to multipoint. When the sender to the recipient at the same time more than one of the same data transmission, it is simply a data packet transmission. In a wireless network, the signal having the natural characteristics of broadcasting. The multicast full advantage of this broadcast feature, reducing the number of transmissions, effectively improving the data transfer efficiency. However, in the cognitive radio network, because there is no access node available channels can be different, the nature of the broadcast may not be well utilized.

In the cognitive radio multi-hop network, the multicast [811] information usually reaches the final destination node via a plurality of intermediate nodes to be forwarded. Therefore, the multicast routing problem had discussed. Existing research has been a lot of multicast routing protocols, then these agreements are based on the traditional wireless networks are not suitable for the new cognitive radio networks. This paper presents a

This work is supported by the National Natural Science Foundation of China under grant number 61262087 and 61262089 distributed multicast routing protocol to solve the problem. This protocol not only to achieve the establishment of the routing process, and also completed the transmission of a node for a reasonable scheduling. To minimize bandwidth consumption during transmission, the paper also presents a new algorithm to achieve node multicast transmission scheduling [10]. Experimental results demonstrate the effectiveness of the proposed protocol and transmission scheduling algorithm.

\section{PROBLEM DESCRIPTION}

Definition 1: a multicast tree refers to a tree for a multicast request established. Root tree node multicast request is issued, and the leaf nodes of the tree is the destination node of the request.

Definition2: Multicast nodes define the bandwidth required for the consumption data to the nodes in the propagation time of one frame so that the number of child nodes of the transmission slot to be.

Definition 3: Whole multicast tree bandwidth consumption can be defined sum of the multicast bandwidth consumption required for all non-multicast tree leaf nodes.

Definition 4: Delay is defined as the establishment of a multicast request multicast tree height multicast requests.

Based on the above definitions, the problem to be solved herein be described in detail below.

Multicast requests having a QoS requirement is known, the request of a given source node $\mathrm{s}$, a destination node $\mathrm{D}, \mathrm{B}$, and a bandwidth demand a delay requirement. In this paper, to solve the problem is: how to build a multicast connection, making this multicast communication bandwidth consumption is minimized while multicast connections on each link can meet the bandwidth needs of B, and the entire multicast latency also no more than delay requirements. In this problem, the bandwidth requirements that are needed for the number of time slots within one frame, and the delay requirement is the maximum number of hops can be allowed.

\section{Multicast Routing Protocol}

From the description of the above problems can know, get to establish minimum bandwidth consumption multicast path, only to be routed, but also to be on the path to the node slot allocation, or transmission scheduling. This section will propose a QoS multicast routing protocol to solve the above problems. The agreement specifies how to establish a multicast connection and how transmission scheduling for a single node. This section first describes the process of the agreement as a 
whole, and further information on transmission scheduling algorithm.

\section{A. Multicast connection establishment}

When the source node sends a QoS multicast request, you must first be able to meet the bandwidth requirements to find a multicast tree for multicast tree and then each non-leaf node for transmission scheduling, reserve the right to its twodimensional slot resources of each link so that the tree can meet the bandwidth requirements. Therefore, in this protocol, the multicast connection establishment is divided into two processes.

\section{B. Forming a multicast tree}

The contribution is a dynamic process using source routing protocol, that is, each data packet or control packet contains all or part of the routing information. When a multicast request arrives, the source node first broadcasts a route list containing their own source routing request packet (Route Request Packet, RREQ) to its neighbors. All nodes receive this packet and will broadcast it further out, and add your own address in the source route list. If a node is not the first time receives the RREQ packet, it will discard the packet directly without multiple broadcasts. This process repeats until the destination node receives the request packet. When the request packet to the destination node, each destination node detects first hops a packet sent over the delay exceeds the limit multicast requests. If more than words, the destination node sends a packet to the source node rejected multicast request will be rejected. Otherwise, the destination node will return a route reply packet (Route Reply Packet, RREP), which contains the route from the source node to the destination node. RREP packet is returned along the packet contains the routing path. When the source node receives the RREP packet to return all of the destination node, the entire process of building a multicast tree is complete. In this case, each intermediate node has been saved for a valid transmission path establishing request.

\section{Resource reservation process}

Reservation process starts from the source node. When the source node to complete the resource reservation, its child nodes begin to reserve allocation of resources. In this way, the process is reserved along the multicast tree, step by step in the direction of the leaf node until all the non-leaf nodes are set aside to complete the process. For a node $\mathrm{u}$, its full reservation process can be described as follows:

1) When a node $u$ need to reserve resources, it sends a Rep Request packet (Request Packet) to all of its child nodes. Rep bandwidth requirements bag contains all recipients (child node) address and a multicast request.

2) For each child node, when it receives Rep package, it will return a response packet Req (Reply Packet) to the node $\mathrm{u}$. Req bag for each child node contains its own two-dimensional slots available.

3) When all of the children nodes collected Req response packet, the node $\mathrm{u}$ is called to reserve a time slot assignment algorithm most suitable for transmitting the two-dimensional slot in future data transfer. The slot allocation algorithm will be described in detail in the next section. If a node $u$ can not successfully reservation data, then the entire multicast request will be canceled. Otherwise, node $u$ will be reserved for slot allocation algorithm determined transmission slot, and broadcasts a reservation packet Res (Reservation Packet) out, notify all nodes within interference range of $u$ already set aside a certain amount of their own the two-dimensional slot in order to prevent these nodes use the same time slot and then to generate interference. Res mainly reserved for packet transmission slot contains reservation information sending node $\mathrm{u}$. Meanwhile, the reservation packet containing twodimensional slots need to be reserved for each recipient corresponding to the information, since the two-dimensional slot for transmitting a reservation, all of the children are not received in this time slot must be .

4) For all nodes Res received the package, if it is not sending a child of a node, then it would Res package set aside two-dimensional slot unusable slots; If it is a child node sending node, then the it should be in the receiving state will own slot is set to receive time slots, while the other set of reserved slots unusable slots. After that, the child node broadcasts a Cfm confirmation packet (Confirm Packet). Confirmation packet contains information about the child node receiving slot reserved. After all the nodes in the interference range of the child node will receive a confirmation packet will receive the package set aside time slots for their unusable slots, thus avoiding the network output interference.

\section{Mulitcast algorithm}

Based on a section of multicast protocols, you can learn to make the entire multicast tree bandwidth consumption minimum, must be such that the bandwidth of each node multicast tree consumption to a minimum. Thus, a key issue to be discussed in this section are: When a non-multicast tree leaf node has received all the children Rep package nodes, that their two-dimensional slots available after the node how transmission schedule, which is reserved for their own transmission slot, so that in the case of a minimum number of reserved transmission slot so that all the children nodes can receive data packets for its transmission.

Specific algorithm, see Algorithm 1. The first 5-9 rows algorithm is used to assign node $\mathrm{u}$ data transmission bandwidth of a minimum number of transmission slots. If your network has enough free slots for node $u$ choose, then this slot allocation algorithm can be successful. Otherwise slot allocation algorithm fails, that node $u$ can not reserve the right to multicast request a two-dimensional slot, then the multicast request will be canceled.

Qos Muticast algorithm

Input: multicast bandwidth requirements B, node and its child nodes u

Output: $\mathrm{u}$ assigned to the node set of two-dimensional slot

1: $S \leftarrow \phi$
2: construct the bipartite graph $\mathrm{G}(\mathrm{X} ; \mathrm{Y} ; \mathrm{E})$
3: while $B \neq \phi$ and $X \neq \phi$ do
4: $B \leftarrow B-1$
5: while $Y \neq \phi$ do
6: $\quad$ pick $x \in X$ that covers the most
remaining uncovered nodes in $Y$




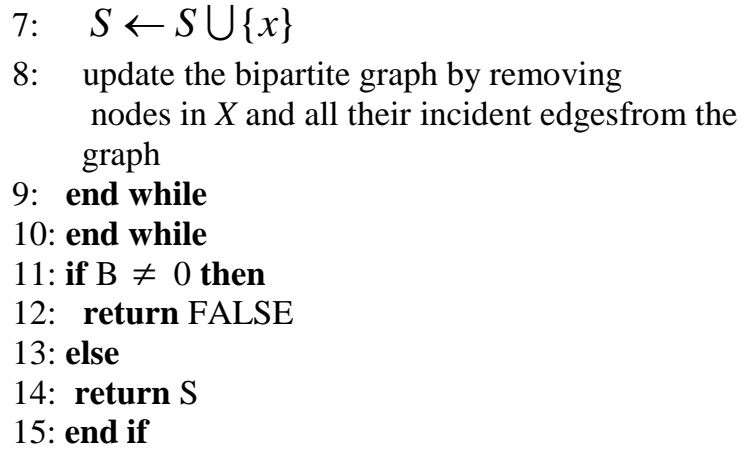

8: $\quad$ update the bipartite graph by removing nodes in $X$ and all their incident edgesfrom the graph

9: end while

10: end while

11: if $B \neq 0$ then

12: return FALSE

13: else

14: return $\mathrm{S}$

15: end if

\section{SIMULATION}

This section analyzes the slot allocation algorithm (multicast slot assignment, MSA) performance. Compare with the traditional algorithm unicast slot allocation algorithm (unicast slot assignment, UNISA). In UNISA algorithm, each node in unicast situation for each child node to transmit data. That is, if a node has $\mathrm{k}$ child nodes, then the node to transmit $\mathrm{k}$ times to make all the kids node receives a data. Assuming multicast tree based on these two algorithms are the same, are established by the method described in this article multicast tree to get.

This section through the detection of the two indicators to measure the performance of MSA algorithm. An indicator is the number assigned to the transmission of the multicast tree is a two-dimensional slots. Another indicator is the success rate of multicast request response. In this paper, the success rate refers to the number of successful response multicast request multicast proportion of the total number of requests.

Simulation experiments performed on the two-dimensional plane with a $100 * 100$. N nodes arranged randomly authorized users and four nodes on the two-dimensional plane. Each authorized user will randomly select a channel as its own channel. According to the characteristics of cognitive radio networks, cognitive radio network node data transfer does not affect the authorized user, so each authorized user of all nodes within interference range are occupied by authorized users can not use this channel. All nodes (including cognitive wireless network nodes and authorized users) transmission radius and interference radius to 25 and 50 respectively. Cognitive radio if and only if the two nodes in the network in the transmission of each other and have a common radius of a data channel between the two nodes to be able to communicate with each other.

UNISA seen from Fig .1 can be used under the same conditions as the algorithm of the required transmission time slot is greater than the number of MSA algorithm. This is because the UNISA algorithm, the multicast tree for each nonleaf nodes have different transmission slots must be reserved for children of different nodes, and MSA proposed algorithm is to make full use of the characteristics broadcasting, making every transmission allow more children nodes simultaneously receive data. As can be seen from Fig .2, the success rate in UNISA MSA algorithm Algorithm. This is because the algorithms required for the transmission time slots is smaller than UNSIA MSA algorithm under the same conditions for all, MSA algorithm chance to successfully transmit slot is reserved for multicast tree, in particular the available timeslots in the network very limited circumstances. Also, the figure can be seen from the demand for bandwidth increases, the success rate of the two algorithms will decline, but the rate of decline of more than UNISA MSA algorithm Algorithm. Particularly when the bandwidth requirement of 7 , when the success rate is substantially 0 UNISA algorithm, and the success rate is still 0.22 MSA algorithm. The reason for this phenomenon is the bandwidth demand grows, the required number of transmission slots UNISA algorithm is much higher than the growth rate of MSA algorithms.

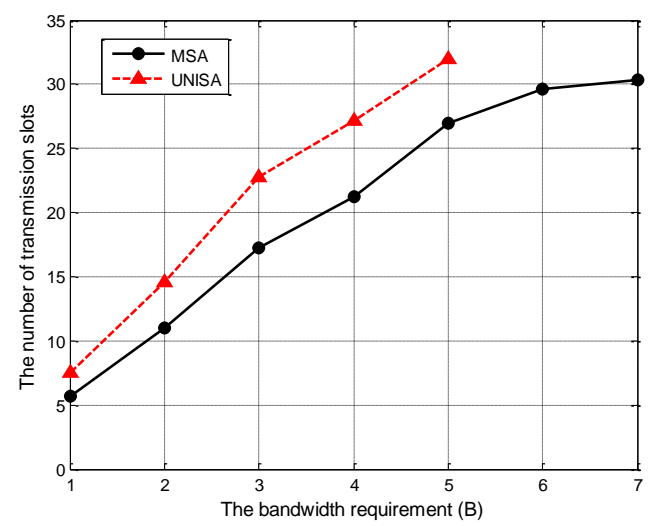

Figure 1. The number of two-dimensional vs slot transmission bandwidth requirements

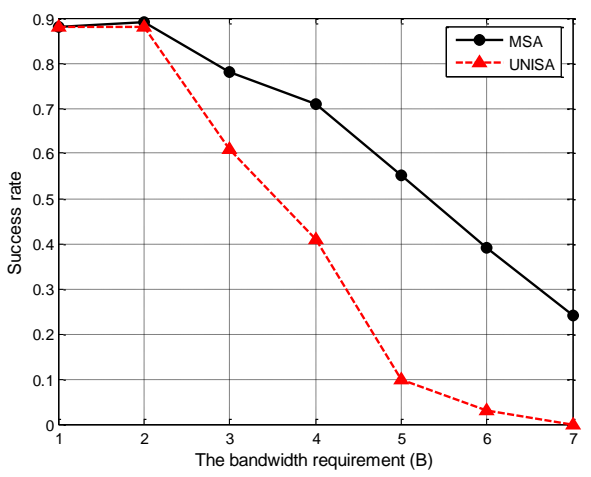

Figure 2. Success rate vs bandwidth requirements

In a second set of experiments, the bandwidth requirements of multicast request is fixed to 3 , and the number of multicast destination node 12 is gradually increased from 1. Other parameter settings and the first set of experiments, the parameter settings consistent. The experimental results shown in Fig .3 and Fig .4. As can be seen from these two photo, when the destination node number is the one when the transmission success rate of the two-dimensional slot and the two algorithms needed multicast request is the same. This is because when the number of nodes for a purpose, when, in fact, a single broadcast, that the two methods come naturally as a result. However, when the number increased when the destination node, the performance of MSA algorithm is significantly better than the UNISA algorithm. Under the same conditions, the number of required MSA algorithm UNISA transmission slot algorithm to be less than, and the success rate of the multicast request to force UNISA higher. And this 
advantage increases with increasing numbers of destination nodes.

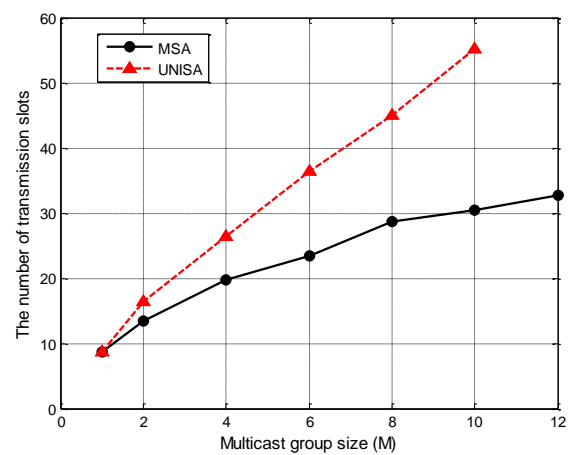

Figure 3. The number of two-dimensional slot transmission vs multicast destination node number

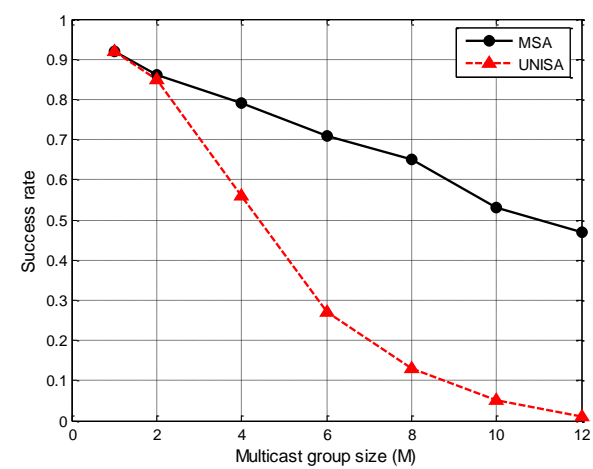

Figure 4. Success rate vs the number of multicast destination node

\section{CONCLUSION}

Due to the heterogeneity of WSN network nodes, in which the multicast network is more complex than in traditional wireless networks. This paper presents a distributed multicast routing protocol. The agreement includes the establishment of a multicast tree and reserve resources process for multicast tree. Through detailed experiments to detect resource reservation algorithm performance, as can be seen from the experimental results proposed transmission scheduling algorithm requires less transmission slots and has a higher success rate in response to multicast requests.

\section{ACKNOWLEDGMENT}

This work is supported by the National Natural Science Foundation of China under grant number 61262087 and 61262089, Key Project of Xinjiang College Teachers Research(Project No. XJEDU2012I09) and Xinjiang University PhD Scientific Research Fund(Project No. BS110127). The authors wish to thank many referees for their suggestions on improving this paper.

\section{REFERENCES}

[1] K. Akkaya and M. Younis, "An Energy-Aware QoS Routing Protocol for Wireless Sensor Networks," in the Proceedings of the IEEE Workshop on Mobile and Wireless Networks (MWN 2003), Providence, Rhode Island, May 2003.

[2] DeyingLi, ZewenLiu, YiHong "Minimum energy multicast/broadcast routing with reception cost in wireless sensor networks", Theoretical Computer Science, vol. 497, no. 4, 2013, pp. 173-180.

[3] S. Lindsey, C. S. Raghavendra, and K. M. Sivalingam, "Data gathering algorithms in sensor networks using energy metrics", IEEE Transactions on Parallel and Distributed Systems, vol. 13, no. 9, Sept. 2002, pp. 924935.

[4] W.R. Heinzelman, A. Chandrakasan, and H. Balakrishnan, "Energyefficient Communication Protocol for Wireless Microsensor Networks", in IEEE Computer Society Proceedings of the Thirty Third Hawaii International Conference on System Sciences HICSS '00), Washington, DC, USA, Jan. 2000, vol. 8, pp. 8020.

[5] Ossama Younis and Sonia Fahmy" Heed: A hybrid, Energy-efficient, Distributed Clustering Approach for Ad-hoc Networks", IEEE Transactions on Mobile Computing, vol. 3, no. 4, Oct.-Dec. 2004, pp. 366-369.

[6] S. Guo, O. Yang. QoS-aware minimum energy multicast tree construction in wireless ad hoc networks. Ad Hoc Neworks (Elsevier), Vol. 2, No. 3, 2004, pp.217-229.

[7] Jiliang Zhou,Qiying Cao,Caixia Li,Runcai Huang. A genetic algorithm based on extended sequence and topology encoding for the multicast protocol in two-tiered WSN, 2010, 30(5):1684-1695.

[8] A. Isazadeh, M. Heydarian. Optimal multicast multichannel routing in computer networks. Computer Communications (Elsevier), Vol. 31, No. 7, 2008, pp.4149-4161.

[9] Chen YS, Lin TH, Lin YW. A hexagonal-tree TDMA-based QoS multicasting protocol for wireless mobile ad hoc networks. Telecommunication Systems, Vol. 35, No. 2, 2007, pp. 1-20.

[10] J. Jia, hang J, Zhang Q. Relay-assisted routing in cognitive radio networks. Proceedings of IEEE ICC, 2009, pp.1-5.. 